\title{
¡Atrevámonos a romper los viejos paradigmas! Desde la universidad y la economía ecológica hacia la consciencia y la integración
}

\author{
Esther Velázquez ${ }^{1}$ \\ Universidad Pablo de Olavide, Sevilla, España. Email: evelalo@upo.es
}

\begin{abstract}
Resumen: En este trabajo comparto una reflexión crítica en torno a los paradigmas que sustentan la educación universitaria y la economía, partiendo de la base de que el paradigma que sustenta a ambas es el mismo, el viejo paradigma newtoniano-cartesiano. Esta toma de consciencia me lleva a unirme a las voces que reclaman la necesidad de romper con estos viejos paradigmas y abrazar uno nuevo, holístico e integrador. Si, como docentes, nos atrevemos a romper los viejos paradigmas; si, como economistas, nos atrevemos a pensar bajo otros enfoques, proponiendo la economía ecológica; y, si como personas, nos reeducamos en la senda de la consciencia y la integración, entonces seremos capaces de transmitir los enfoques y valores del nuevo paradigma.
\end{abstract}

Palabras clave: Paradigmas, universidad, economía ecológica, consciencia, integración.

\section{Let us dare to break old paradigms! From university and ecological economics towards awareness and integration}

Abstract: A critical consideration about paradigms that support higher education and economy is shared in this paper, starting out from the awarenes that the paradigm that supports both is the same one, the old newtonian-cartesian. This awareness leads me to join the voices calling for the need to break with these old paradigms and to look at a new one, holistic and integrative. If, as teachers, we dare to break old paradigms; if, as economists, we dare to think under other approaches, proposing the ecological economics; and if as persons, we re-educate ourselves on the path of awareness and integration, then we will be able to teach the values_and approaches of the new paradigm.

Key words: Paradigms, university, ecological economics, awareness, integration.

\section{Vamos ousar quebrar velhos paradigmas! Desde a faculdade e economia ecológica para a consciência e integração}

Resumo: Neste artigo vou compartilhar uma reflexão crítica sobre os paradigmas que sustentam o ensino superior ea economia, sobre a base do paradigma que suporta ambos é o mesmo, o velho paradigma newtoniano-cartesiano. Esta 
constatação leva-me a juntar as vozes que pedem a necessidade de quebrar os nossos velhos paradigmas e abraçar umo novo, holístico e inclusivo. Se, como professores, nos atrevemos a quebrar velhos paradigmas, se, como economistas ousamos pensar em outras abordagens propondo uma economia ecológica, e se como pessoas reeducar-nos no caminho da consciência e da integração, em seguida, seremos capaz de transmitir os valores e abordagens dos novos paradigmas.

Palavras-chave: Paradigmas, Universidade, Economia Ecológica, consciência, integração.

\section{Introducción}

En los tiempos que corren, donde tanto se habla de la educación, de la universidad, de la enseñanza, me gustaría compartir una reflexión desde el espacio en el que me muevo: la universidad pública y las enseñanzas de economía. Pero no voy a hablar de la tan necesaria enseñanza pública, de calidad y democrática que requerimos en estos turbulentos momentos; no voy a hablar tampoco de las enseñanzas de economía en ninguna universidad concreta; por el contrario, mi propósito no es otro que compartir una reflexión crítica en torno a los paradigmas que sustentan una y otra: educación universitaria y economía. No obstante, este es el primer acercamiento que hago al tema y así me gustaría que fuera entendido: una primera reflexión con la que no trato de cerrar ningún debate sino, por el contrario, participar en uno ya abierto para seguir avanzando por este camino, que no es más que el de mi propia evolución como ser humano.

Tal como afirman Barkin y Elizalde (2012) “existe un creciente consenso en el mundo académico respecto a que el paradigma que ha inspirado el surgimiento del mundo moderno enfrenta una profunda crisis (y que desde) la vida académica e intelectual es imprescindible proponer nuevos paradigmas que trasciendan las disciplinas que han evolucionado para respaldar el sistema actual”. Con esta idea como telón de fondo, me atrevo a compartir esta reflexión crítica con la ilusión de que sea un paso más el camino iniciado.

\section{Viejos paradigmas: ¿Dejándonos llevar por la inconsciencia o una inconsciencia muy consciente?}

Max-Neef (2005), en su artículo "Del saber al comprender: navegaciones y regresos", hace un magnífico recorrido por las valiosas aportaciones de renombrados físicos, filósofos y pensadores para demostrar que el paradigma dominante es el producto de las elecciones que hicimos en el pasado como civilización occidental. Así, elegimos el "temor" de Maquiavello frente al "amor" de Francisco de Asís; la "verdad absoluta” de Bacon antes que las "verdades múltiples” de Giovanni Pico Della Mirándola; le dimos prioridad al "mecanicismo y al reduccionismo" de Descar- 
tes, ignorando los aportes a la visión "holística” de Giordano Bruno; y por último, nos quedamos con la "razón” de Newton y Galileo antes que con el "sentimiento y la intuición” de Goethe. Estas elecciones, han contribuido a configurar la civilización occidental y, de la misma forma, han condicionado particularmente la configuración de la universidad, de la docencia universitaria en general, y de la económica en particular.

A poco que nos fijemos y nos atrevamos a ver lo que tenemos delante, podemos afirmar sin miedo a equivocarnos que vivimos una esperpéntica paradoja en lo que a la universidad y a la docencia en economía se refiere. La universidad, supuestamente una institución creadora de pensamiento libreque debería cumplir un relevante papel como espacio para la transformación social, puede convertirse en una plataforma de promoción del pensamiento único. Y la enseñanza en economía no escapa a dicha limitación, pues en muchos supuestos ésta se restringe a los principios y fundamentos de la economía neoclásica. Recientemente he tomado consciencia de que estas limitaciones que constriñen a una y a otra (los males que nos aquejan a nosotros mismos, en definitiva) se deben a que ambas responden a un mismo paradigma: las viejas ideas cartesianas-newtonianas. Ideas extraordinarias en su tiempo pero que hoy hay que tomarlas con toda la precaución necesaria si queremos entender la realidad del siglo XXI, diversa y compleja.

Comenzando por Descartes (1637), las ideas de su Discurso del Método que nos interesan resaltar son, por un lado, la razón pura, a partir de la cual el conocimiento avanza a golpe de cuantificación matemática, dejando de lado, ignorando y olvidando, todo aquello que no se puede modelizar matemáticamente. Y por otro, la segunda regla del Discurso a partir de la cual las dificultades a examinar serán divididas en tantas partes como fuere posible para poder encontrar así la mejor solución.

Por su lado, las leyes de la mecánica postuladas por Newton (publicadas en 1687) refuerzan esta idea de parcelación y descontextualización, enunciandola conocida frase"el todo es explicado por la suma de las partes”, y encaja a la perfección con las matemáticas como herramienta de razonamiento postuladas por Descartes. En esta línea de pensamiento, poco antes, Galileo (1623) había rescatado la idea de "el libro de la naturaleza"en la que afirmaba que "la filosofía está escrita en ese libro enorme que tenemos continuamente abierto delante de nuestros ojos (hablo del universo), pero que no puede entenderse si no aprendemos primero a comprender la lengua y a conocer los caracteres con que se ha escrito. Está escrito en lengua matemática, y los caracteres son triángulos, círculos y otras figuras geométricas sin los cuales es humanamente imposible entender una palabra; sin ellos se deambula en vano por un laberinto oscuro" (Galileo, 1623: 6; citado en Calvino, 2009: 49).

De esta manera, "la economía moderna (...) ve la realidad como un conjunto de objetos inertes, sometidos a leyes mecánicas y lineales, y tiende a oponer a los seres humanos contra la naturaleza y entre si. Los econo- 
mistas clásicos, desde Adam Smith hasta Vilfredo Pareto, tomaban como modelo la física de Newton, cuyos presupuestos siguen dominando la economía aunque estén superados desde hace un siglo" (Pigem, 2010: 31). En efecto, estas ideas (razón pura, matematización, parcelación de la realidad, descontextualización) arraigaron en el "pensar" económico y en la docencia de la (que me voy a permitir llamar) vieja economía, de la economía neoclásica; tanto así que la economía que se estudia, la que se enseña y la que se aplica es una economía matematizada, y como tal, cuantitativa, pretendidamente positiva, descontextualizada y parcelada. Y así, "el pensamiento va paulatinamente adoptando un enfoque atomista y procura aislar los elementos del universo observado (de tal manera que) la preocupación se centra en el estudio de las propiedades de cada uno de los elementos y no de las relaciones que existían entre ellos” (Aguilera, 1996: 8). Y no sólo se parcela, sino que el objeto de estudio de la economía se reduce y se cierra hasta dejar fuera de su ámbito de estudio todo aquello que no es directamente útil para el ser humano, que además se pueda producir y apropiar por éste y que pueda ser valorado monetariamente para así poderlo incluir en la lógica del mercado (Naredo, 1987).

Este sistema cerrado así configurado, matematizado y monetarizado, es respaldado por otro gran avance de la ciencia: el primer principio de la termodinámica (enunciado por Joule, 1845), también conocido como ley de la conservación de la energía, por la que "la energía ni se crea ni se destruye sino que se transforma". Este principio pone el acento en la "cantidad” de energía (y no en la calidad), primando pues la "cuantificación” e ignorando todo aquello que no pueda ser cuantificado (y matematizado). Esta idea de primar la cuantificación es recogida por la ciencia económica, y así se relegan a un segundo plano (o se ignoran) los aspectos cualitativos de los procesos económico y sociales. De esta forma, se cierra el círculo económico sobre estas ideas físicas y filosóficas, sobre la razón científica y objetiva, pretendidamente positiva por estar basada en una cuantificación monetaria en base a modelos matemáticos y, así, supuestamente libre de valores.

Esta es la economía que se enseña hoy (me atrevería a decir que) en todas las facultades. Es tan evidente que esta economía no funciona (o mejor dicho, funciona únicamente para unos pocos, y no funciona para la mayoría) que hay autores como Max-Neef (2012) que afirman: "Estoy convencido de que no podremos mejorar las condiciones mundiales mientras continúen las universidades del mundo enseñando esta economía. Las universidades tienen una responsabilidad gigantesca de la que parecen no tomar conciencia”. Llegados a este punto, me pregunto si vivimos una inconsciencia generalizada en cuanto a los paradigmas subyacentes a nuestros pensamientos, actos y sentimientos; o si, por el contrario, asistimos a un proceso de inconsciencia muy consciente.

Para alcanzar esa consciencia de la que nos habla Max-Neef, hemos de darnos cuenta que estos viejos paradigmas también han configurado la universidad actual. En efecto, la universidad del siglo XXI recorre los ca- 
minos de la ciencia, legitimada por una razón entendida como antagónica a la intuición-emoción, que nos lleva a la parcelación del conocimiento y a lo que Morin (2001)ha llamado la hiper-especialización, "departamentalizando” y “disciplinando” la universidad (Max-Neef, 2004). Esta universidad, así entendida, se supone que transmite un saber "científico”, un saber "razonado y razonable", cuando en realidad lo que se transmite es una acumulación de conocimientos parcelados y compartimentados derivados de una especialización que abstrae (Max-Neef, 2005); derivados de una disciplina compartimentada "cuyas fronteras rompen arbitrariamente la sistematicidad (la relación de una parte con el todo) y la multidimensionalidad de los fenómenos; (consiguiendo únicamente transmitir un conocimiento de la realidad) determinista, mecanicista, cuantitativo y formalista (que) ignora, oculta o disuelve todo lo que es subjetivo, afectivo, libre y creador" (Morin, 1993: 66, 68). Como nos advierte Feyerabend (1975: 153), "un río puede estar subdividido por fronteras nacionales, pero esto no lo convierte en una entidad discontinua”.

Así hemos llegado “a un punto en nuestra evolución humana, caracterizado por el hecho de que sabemos mucho pero comprendemos poco" (Max-Neef, 2005: 3). Es más, podríamos incluso afirmar que la educación para comprender está ausente de nuestras enseñanzas, llegando Aldana (2007: 60) a preguntarse "¿a quienes conviene que sepamos mucho pero que comprendamos poco?”. Curiosamente, muchos años antes, Aguilera (1996) respondía a esta pregunta de una manera lúcida, "el problema consiste en que enseñados y acostumbrados a pensar bajo las premisas dominantes, dar el paso de atreverse a pensar bajo las premisas alternativas no es nada fácil (...) porque exige romper las barreras departa-mentales y disciplinares para pensar en términos trans-disciplinares, algo que supone, además de un atrevido reto intelectual, un reto a los intereses académicos establecidos".

Hay un abismo entre el saber y el comprender, ya que "sólo podemos pretender comprender aquello de lo que nos hacemos parte (...); el comprender es el resultado de la integración, mientras que el saber ha sido el resultado de la separación (...); el comprender es holístico, mientras que el saber es fragmentado” (Max-Neef, 2005: 4); el saber deriva de la razón y el comprender de la razón-emoción. Así, convendrá conmigo el lector que en la universidad del siglo XXI se ofrece un saber que nos aleja del comprender; y si el comprender es integración, hemos de concluir que este tipo de "saber" es disociación, pudiendo pues afirmarse que la universidad actual podría estar contribuyendo a la disociación antes que a la integración (Aguilera: 2010).

Y esta transmisión, únicamente, del “saber” científico sólo es posible si la actividad docencia-discencia lo permite. Como bien afirma Freire (1997: 25) "no hay docencia sin discencia (esto es), quien enseña aprende al enseñar y quien aprende enseña al aprender”. Por lo tanto, docencia y discencia son las dos caras de una misma moneda; como sea la una, así será la otra, y viceversa. 
Con relación a la discencia, nuestra forma de aprender, podemos afirmar que todos aprendemos mediante el "libre ejercicio de la facultad más expandida y más viva durante la infancia y la adolescencia: la curiosidad; (no obstante, ésta está) muy a menudo sofocada por la instrucción, cuando por el contrario se trataría de estimularla o, si está dormida, despertarla” (Morin, 2001: 48). De esta manera, a los seres humanos se les educa "para amoldarse, para encajar dentro de la sociedad (...) para adaptarse a la corriente de la actividad social y económica” (Krishnamurti, 1974: 16); y este proceso genera "un desprecio por el auténtico conocimiento, por la labor de creación y de producción que uno mismo puede realizar” (Delval, 1990); y así vamos fomentando el desarrollo de personas inseguras y temerosas, no conocedoras de su propio potencial intelectual e intuitivo. Ortega (1930: 91) refleja esta idea a la perfección al preguntarse “¿Cuántos por ventura son los afortunados que al salir de los años de educación llevan hincada en su conciencia la idea de que, fuertes o débiles, mejor o peor dotados, encierran en su ser la posibilidad delicadísima de algo nuevo, bello y fecundo, y que su vida debe ser para ellos, el más armonioso espectáculo y el más valiente experimento?”.

Hay algo que ayuda en todo este proceso de adormecimiento del estudiante: elmanualque "contiene el adoctrinamiento envasado” (Aguilera, 2010).En efecto, el manual, lejos de fomentar la curiosidad del estudiante para que pueda plantearse sus propias preguntas, obliga a responder las preguntas de otros, que con bastante frecuencia, a su vez, repiten las preguntas previas que a ellos les formularon. Así, la “función del alumno es tratar de reproducir lo más fielmente posible lo que dice la autoridad sin desviarse; (por lo que podríamos decir que) aprender es entonces un acto de sumisión a la autoridad” (Delval, 1990). Pero esto no es nuevo, pues cuentan que "las autoridades escolares no toleraban que el pequeño Einstein se parase a reflexionar sobre el significado de las preguntas de los profesores, obligándole a responder con inmediatez” (Calaprice, 2002: 70). En efecto, la "inmediatez"es irreflexión, es prejuicio, es obediencia, es sumisión, es el fomento de la competitividad más insana... pero podría ser exactamente esto lo que estuviéramos fomentando y premiando desde la más tierna infancia, y evaluando y premiando en la universidad.

Y si preocupante es el hecho de basar la docencia universitaria (me refiero fundamentalmente en las facultades de economía) en los manuales, algo tan preocupante o más que esto es el hecho de que hoy (curso académico 2012-13) siguen estando al uso los mismo manuales, no de 10 años atrás, sino de 20 y 30. Manuales que no han movido un ápice sus teorías y obsoletos paradigmas. Cualquiera podría pensar que, tras la fortísima crisis por la que atravesamos y la debacle acontecida que ha puesto en evidencia la insolvencia de los paradigmas actuales, cambiarían radicalmente los manuales que se ofrecen. Muy al contrario, no únicamente no se han modificado, sino que muchos de los profesores de hoy siguen explicando las mismas teorías del libre mercado y de la competencia perfecta sin ningún tipo de cuestionamiento ni rubor. La excesiva dependencia de los manuales, y de los profesores que imparten estas materias basadas en viejos 
paradigmas, impide a los estudiantes adquirir un conocimiento crítico que les lleve al cuestionamiento de los paradigmas actuales. De esta manera, "la universidad ha dejado de enseñar a pensar (...) y se centra cada vez más en enseñar a obedecer” (Aguilera, 2010: 6). Y todo ello a pesar de que un economista prestigioso como Coase (1998:72) afirmara tiempo atrás que "la economía convencional, por lo que veo en las revistas científicas, en los manuales y en los cursos que se enseñan en los departamentos de economía se ha convertido en algo cada vez más abstracto y, aunque se propone lo contrario, de hecho está poco preocupada por lo que ocurre en el mundo real”.

Los estudiantes que aceptan un proceso basado en estos viejos paradigmas son los principales perjudicados, y con ellos toda la sociedad, pues "la economía, tal como se enseña, se convierte (...) en una parte de la maquinaria mediante la cual se impide al ciudadano o al estudiante ver de qué manera está siendo gobernado o habrá de estarlo” (Galbraith, 1982: 189). Como dice Kozy (2009), "la cuestión es que una vez que una persona adopta una ideología, pocas veces la cuestiona (...) El fallo, claro está, reside en que la educación no alienta el cuestionamiento de la ortodoxia”.

Y si esto no es suficientemente evidente y preocupante, me preocupa más si cabe que "la sociedad contemporánea parezca (...) tan satisfecha de sus centros de enseñanza superior (...) revelando (...) que la gente no quiere sabiduría sino recetas” (Ortega y Gasset, 1930: 89) (palabras de Ortega de 1930 y que siguen estando de plena actualidad); unas recetas que le permitan la adaptación al sistema sin mayores complicaciones. Es más, muchas veces es el propio estudiante, desconocedor de su potencial, quien demanda una formación supuestamente técnica y científica, que le requiere el mínimo esfuerzo y que le capacita para el mundo laboral. De esta manera, "la educación no está al servicio de la evolución humana sino de la producción” (Naranjo, 2008: 78), fomentando el aprendizaje de un modo de pensar rutinario, a base de la creación de hábitos que permitan obtener un empleo (Krishnamurti, 1974: 150). Podríamos pues decir quelas elecciones del estudiante universitario (aunque, afortunadamente, no de todos) están demasiado condicionadas por la necesidad futura de encontrar un empleo en el "mercado", lo que automatiza su aprendizaje, le conmina a obedecer con inmediatez y anula su capacidad de pensar, su intuición y su emoción, su potencial para desarrollar una actitud crítica y su propia evolución como ser humano.

Paralelamente a este proceso discente, se desarrolla el proceso docente. No obstante, recordemos como dice Freire (1997: 25) que no hay docencia sin discencia, por lo que el proceso no es tanto de ida y vuelta sino más bien un bucle que se retroalimenta ya que "quien forma se forma y re-forma al formar y quien es formado se forma y forma al ser formado”. Con relación a este proceso docente, algunos profesores (aunque, igual que ocurre con los estudiantes, afortunadamente se ven indicios de cambio), "los profesores frecuentemente están mucho más preocupados por la formación de hábitos y rutinas que por entender lo que se está haciendo” 
(Delval, 1990). Esto es, podría estar sucediendo que los estudiantes aprendan hábitos rutinarios de pensamiento porque los profesores estamos preocupados porque así sea: “ ¿a (...) qué profesores les interesa que los estudiantes piensen de verdad por su cuenta? ¿Por qué se sienten tan agraviados los profesores cuando un estudiante se atreve a mostrar que piensa por su cuenta, más allá de los ejercicios teóricos de pizarra?” (Aguilera, 2010: 7).

En este sentido, tal vez esté influyendo de manera decisiva, entre otros factores, la clara esquizofrenia que se vive en el seno de la universidad española, donde la función principal del profesor, como tal profesor, ha de ser "educar" (y no únicamente "enseñar); y, no obstante, sus posibilidades de promoción dependen de su actividad investigadora y, fundamentalmente (por no decir, casi en exclusividad) por las publicaciones "científicas” realizadas. Podría dar muchos y variados ejemplos de denuncias sobre el estúpido e inservible sistema de publicaciones académicas, y con él, el propio sistema de evaluación y promoción del profesorado en España. No obstante, me quedo con una frase de Ortega (1930: 74) realizada en los años treinta del pasado siglo: "no se decidirá en la elección del profesorado el rango que como investigador posee el candidato, sino su talento sintético y sus dotes de profesor”; ¿Tendrá alguna razón Berzosa (2006) cuando afirma que con el actual sistema de selección "se prescinde de gran parte de buen profesorado, dando cabida a otros muchos que tendrán méritos muy estimables y valorables, y que hay que apoyar sin lugar a dudas, pero que no son buenos docentes de los que estamos tan necesitados?”. No estoy queriendo decir con esto que el profesor universitario no haya de investigar; muy al contrario, comparto con Freire (1997: 30), que "la indagación, la búsqueda, la investigación, forman parte de la naturaleza de la práctica docente. Lo que se necesita es que el profesor, en su formación permanente, se perciba y se asuma, por ser profesor, como investigador”. En otras palabras, tendríamos que entender la docencia universitaria y la investigación desde la integración de ambas, pero no darle prioridad a ésta última en detrimento de una buena docencia, como fomenta el actual sistema de promoción del profesorado universitario. Claro que esto es suponer que los poderes económicos no tienen un control sobre las cada vez más concentradas editoras de revistas científicas y los índices de impacto que a la postre pretenden juzgar la calidad de las aportaciones científicas, materia que precisaría de un estudio monográfico.

Resumiendo, podríamos decir que la universidad del siglo XXI podría estar avanzando por una senda en la que se transmite un saber, como un conjunto de conocimientos científicos, objetivos, transmitidos de manera parcelaria e hiper-especializada; donde el estudiante "ideal” se podría caracterizar por ser una persona sumisa, obediente, con una fuerte inseguridad y desconfianza en su propio potencial, quien no se permite pensar por sí misma, y a quien se le niega la intuición y la emoción; y este estudiante estaría fomentado por un docente, igualmente obediente, igualmente inseguro y desconfiando de sí mismo. Una docencia centrada en formar "recursos humanos", ante que personas integradas. Y todo ello, seamos o no conscientes, a partir de la aceptación deun paradigma cartesiano-newtoniano, 
en el que la razón y el temor imponen una verdad absoluta que fomenta el individualismo y la competitividad.

\section{El Nuevo Paradigma Holístico e Integrador: universidad y economía ecológica}

Partiendo de la idea de paradigma científico de Kuhn (1962), por el que éste se entiende como el conjunto de creencias y compromisos de una comunidad científica capaz de explicar la realidad en un momento determinado, podemos afirmar que asistimos a una crisis epistemológica en la que "el paradigma, en lugar de ser una norma silenciosa, casi invisible, en lugar de estar 'por encima de todo', es discutido y cuestionado. Los miembros de la comunidad, en vez de trabajar con unanimidad en resolver los problemas reconocidos por todos, plantean cuestiones 'fundamentales', cuestionan la legitimidad de sus métodos. El grupo, que la educación había hecho homogéneo en lo que se refiere al trabajo de investigación, se diversifica, las diferencias de puntos de vista, de experiencias culturales, de convicciones filosóficas, toman luz y juegan a menudo un papel decisivo en el descubrimiento del nuevo paradigma. La aparición de este último acrecienta aún más las discusiones (...) hasta que una diferencia, amplificada y estabilizada por los circuitos académicos, decide la victoria de uno de ellos" (Prigogine y Stengers, 1983: 316, 317).

Tal y como afirman Barkin y Elizalde (2012) "hay muchas búsquedas iniciadas para encontrar estos nuevos caminos (...) y es interesante constatar que muchas de las pistas posibles de seguir se encuentran también entre los paradigmas heterodoxos de las ciencias sociales. Muchos de ellos largamente despreciados y desvalorados desde el pensamiento ortodoxo”. Estamos en ese momento en el que la aparición del nuevo paradigma acrecienta las discusiones; estamos en el momento en el que se impone la necesidad de que el viejo paradigma newtoniano-cartesiano, tan lúcido en su momento (siglo XVII), sea superado por un nuevo paradigma que ayude a entender el mundo y la sociedad en la que vivimos al inicio del siglo XXI; un nuevo paradigma que venga de la mano de "la necesaria cosmovisión holística que se caracteriza por la integración de diversas escuelas así como diferentes tradiciones espirituales” (González Garza, 2009: 40).

Desde la economía se han planteado nuevas alternativas, despreciadas y desvaloradas por los economistas neoclásicos, aunque es ya bien conocido que el problema de la economía (neoclásica) no es que naciera a la luz de los paradigmas imperantes en su tiempo, sino que quedó anclada en aquellos, mientras el resto del mundo evolucionaba. En efecto, la física clásica y sus leyes de la mecánica fueron completadas por la teoría de la relatividad de Einstein (1905), donde el tiempo y el espacio, esto es la contextualización y la escala, adquieren un papel esencial; donde todo es un continuo que ha de ser analizado como tal, y no a través de sus partes. 
Sin embargo, y aunque "la relatividad ha modificado el concepto clásico de objetividad física, (puede considerarse que) es aún una continuación de la física clásica (en el sentido que) puede concebir un matemático supremo que, como Einstein argumentaba, (...) conocía la fórmula del Universo a partir de la cual todos los posibles puntos de vista del mundo podrían ser derivados. La mecánica cuántica, por otra parte, es la primera teoría física que ha roto verdaderamente con el pasado” (Prigogine, Stengers, 1983: 251). Y será el principio de incertidumbre enunciado por Heisenberg en 1927 el que marcará una diferencia clara entre la física clásica y la física cuántica (Prigogine, 1983: 13), con importantes repercusiones tanto en la filosofía como en las propias ciencias sociales. En efecto, las ciencias sociales en general, y la economía ecológica en concreto (como veremos a continuación), incorpora el concepto de ciencia postnormal (Funtowicz y Ravetz, 1993) donde la incertidumbre es la base de los procesos económicos y sociales y en los que ha de imperar el principio de precaución.

Por otro lado, la ley de la entropía (Clausius, 1850) introduce el concepto de irreversibilidad de los procesos físicos, y "la termodinámica de los procesos irreversibles ha descubierto que los flujos que atraviesan ciertos sistemas físico-químicos y los alejan del equilibrio pueden alimentar fenómenos de auto-organización espontánea, rupturas de simetría, evoluciones hacia una complejidad y una diversidad crecientes. Ahí donde se paran las leyes generales de la termodinámica puede revelarse el papel constructivo de la irreversibilidad" (Prigogine y Stengers, 1983: 299). Esto es, se introduce la idea del caos creativo mediante procesos puramente azarosos.

Mediante este rápido vistazo a la evolución de la física y de la ciencia podemos apreciar el salto cualitativo en la forma de entender el mundo y la realidad; forma que quedó completamente ajena a la evolución de la economía como “ciencia”.Pero la evolución no se limitó a la física. Así, la racionalidad cartesiana ha sido ampliamente criticada por filósofos y pensadores, llegando Feyerabend (1975, 167, destacado en el original) ha afirmar que "Dada la ciencia, la razón no puede ser universal y no puede excluirse la sinrazón”. De igual manera, las ideas de Goethe sobre la emoción y la intuición, hasta las más recientes de Damasio (1994), Max-Neef (2004, 2005, 2012), Morin (1993, 2001), Krishnamurti (1974), Funtowicz (1993), etc. van quedando relegadas, ignoradas, hasta ser olvidadas.

Sin embargo, hay otras corrientes de pensamiento que recogen estas lúcidas ideas y tratan de avanzar con ellas. La Economía Ecológica se configura como un avance en esta dirección, definiéndose como "una forma alternativa de comprender la realidad, no sólo el proceso económico, desde una visión sistémica, transdisciplinar y coevolutiva, en la que la dimensión física, los valores, las reglas del juego y la intuición adquieren un papel predominante” (Asociación economía ecológica en España, 2011: 3). De esta manera, se rompe con el antiguo paradigma newtoniano-cartesiano, para proponer un nuevo paradigma holístico e integrador. 
Aquí tenemos, pues, la ruptura con Descartes y Newton como forma de entender la realidad. Este nuevo paradigma requiriere una visión sistémica, donde las partes no pueden ser separadas, y donde las relaciones dinámicas (coevolutivas) entre esas partes son fundamentales para la comprensión.

Este paradigma holístico e integrador nos llevaría a impartir una docencia en economía ecológica, en lugar de economía neoclásica, en una universidad donde la razón compartiera espacio con la emoción y la intuición; en la que se aceptara la posibilidad y la existencia de verdades múltiples y donde la colectividad y la cooperación predominaran por encima de la individualidad y la competitividad. Bajo este nuevo paradigma, el docente acompañaría al estudiante en su evolución personal; realizaría una docencia “empoderándolo" (González, 2013), ayudándole a descubrir el sabio que lleva dentro, al mismo tiempo que recorre su propio camino de evolución. Acompañaría al estudiante en su comprender, más allá del conocimiento científico que éste fuera adquiriendo; se le permitiría al estudiante que pensara y sintiera en libertad, lejos del adoctrinamiento de los manuales, de tal forma que pudiera descubrir sus propias preguntas. El docente transmitiría algo más que pura y razonada ciencia, transmitiría cultura en su acepción más amplia (Ortega y Gasset, 1930), reconociendo un conocimiento subjetivo, transdisciplinar, holístico y donde la incertidumbre tiene un espacio destacado. De esta manera, el estudiante desarrollaría una afianzada seguridad y confianza en sí mismo que le permitiría desarrollar su curiosidad y su creatividad. En definitiva, siguiendo el nuevo paradigma holístico-integrador, tanto en la visión y constitución de la universidad como en la enseñanza de una economía ecológica, el docente y el estudiante se reforzarían mutuamente a través del proceso de educación- reeducación, y se acompañarían en sus respectivos procesos de evolución.

\section{Desde la universidad y la economía ecológica hacia la toma de consciencia y la integración}

Tras esta reflexión, llego al convencimiento de que hemos de romper con los viejos paradigmas, no únicamente en la universidad (fomentando una universidad en pro del holismo y la integración), no únicamente en la enseñanza de la economía y en la propia economía (trabajando por una economía ecológica, sistémica y coevolutiva), sino que hemos de permitirnos evolucionar en la senda de la consciencia y de la integración; para ello es importante que seamos capaces de preguntarnos ¿qué persona somos, dónde estamos y qué podemos hacer? Necesitamos una "re-educación" personal "que incluya prácticas concretas que sirvan al cultivo de la mente profunda y un proceso de autoconocimiento guiado que lleve no sólo a cambios de conducta sino a esa transformación que es la esencia de la maduración propiamente humana” (Naranjo, 2010: 144). Como docentes tenemos una importante responsabilidad en la deriva de la universidad (como nos recordaba Max-Neef), de la economía, de nuestra propia evolución y 
de la de los estudiantes. Habríamos de considerar muy seriamente la recomendación de la UNESCO (II Congreso Holístico Internacional, Belo Horizonte 1991) por la que se nos insta a "tomar en cuenta la urgencia de incorporar el factor emocional y espiritual a la educación”.

$\mathrm{Si}$, como docentes, nos atrevemos a abrazar los nuevos paradigmas; si, como economistas, nos atrevemos a pensar bajo otros enfoques; $y$, si como personas, nos reeducamos en la senda de la consciencia y la integración, entonces seremos capaces de transmitir los enfoques y valores de los nuevos paradigmas; si nos acomodamos en los viejos y nos dejamos llevar por la inercia académica, ahí nos quedaremos anclados, como la vieja economía.

Comparto con Naranjo $(2010,194)$ que "la educación para la libre realización de nuestras potencialidades evolutivas y creativas puede ser crítica para nuestra supervivencia colectiva”. Así, para fomentar una educación de estas características, hace falta una "re-educación” de los docentes en este sentido. Sin nuestra propia "re-educación”, el camino difícilmente cambiaría de sentido. Atrevámonos, pues,a romper los viejos paradigmas; de esta manera pasaremos de una inconsciencia consentida a una consciencia integrada. 


\section{Nota}

${ }^{1}$ La autora agradece las aportaciones realizadas por el evaluador anónimo del trabajo original, así como los comentarios y sugerencias de Federico Aguilera, Francisco Puche y Jordi Pigem. 


\section{Bibliografía}

Aguilera, F. (1996), “Economía y medio ambiente: un estado de la cuestión”. Grandes cuestiones de la economía, 10. Fundación Argentaria, Madrid.

Ídem (2010), “La economía como sistema abierto. De la disociación a la integración”. Revista Sustentabilidad(es), 2: 28-49.

Aldana, C. (2007), La revolución del sentir. Nuestro derecho a transformar el mundo. FundaciónIntermón Oxfam, Madrid.

Barkin, D. y Elizalde, A. (2012), “Hacia la construcción de un nuevo paradigma social”. Polis, 33/2012. (Consultado en diciembre 2012, http:// polis.revues.org/8398).

Berzosa, C. (2006), “Elogio a la docencia universitaria”. Publicado en El País, 08-05-2006.

Calaprice, A. (2002), Querido profesor Einstein. Gedisa Ed. Barcelona.

Calvino, I. (2009), “El libro de la Naturaleza en Galileo”. Ciencias, 95: 50-53.

Coase, R. (1998), “The new institutionaleconomics”. American EconomicReview, 88 (2): 72-74.

Damasio, A. (1994), El error de Descartes. La emoción, la razón y el cerebro humano. Ed. Crítica, Barcelona. (Ed. 2010).

Delval, J. (1990), “La destrucción del conocimiento”. Publicado en El País, 16-01-1990.

Descartes, R. (1637), El discurso del método. Revista de Occidente, Madrid. (Ed. 1974).

Freire, P. (1997), Pedagogía de la autonomía. Ed. Siglo XXI, Madrid.

Funtowicz, S. y Ravetz, J. (1993), Epistemología Política. Ciencia con la gente. Centro Editor de América Latina. Buenos Aires.

Galbraith, J. K. (1982), Memorias. Una vida de nuestro tiempo. Grijalbo, Madrid.

Galileo, Galilei (1623), II Saggiatore. Liber (consultado en febrero 2013, http://www.liberliber.it/mediateca/libri/g/galilei/il_saggiatore/pdf/ il_sag_p.pdf. Versión utilizada The Essayer, consultado en http:// www.stanford.edu/ jsabol/certainty/readings/Galileo-Assayer.pdf). 
González, C. (2013), Veintitrés maestros con corazón. Un salto cuántico en la enseñanza. (Consultado en febrero2013, http:// sindamel.files.wordpress.com/2010/02/veintitresmaestrosdecorazon.pdf)

González Garza, A. M. (2008), Educación holística. La pedagogía del siglo XXI. Ed. Kairós, Barcelona (Ed. 2009).

Kuhn, T. S. (1962), La estructura de las revoluciones científicas. Fondo de Cultura Económica, México (Ed. 1971).

Krishnamurti, J. (1974), Sobre la educación. Editorial Kairós, Barcelona. (Ed. 2009).

Kozy, J. (2009), “El error de la oferta y la demanda”. Rebelión. (Consultado en abril 2009, http://www.rebelion.org/noticia.php?id=83579).

Max-Neef, M. (2004), Fundamentos de la transdisciplinariedad. Universidad Austral de Chile.

Ídem (2005), Del saber al comprender: navegaciones y regresos. Conferencia impartida en la Universidad Militar Nueva Granada, Colombia.

Ídem (2012), "Por una enseñanza no tóxica de la economía”. Actualidad. La Revista de la Universidad Austral de Chile. (Consultado en diciembre 2012,http://actualidad.uach.cl/?p=845).

Morin, E. (1993), “El desafío de la globalidad”. Archipiélago, 16: 66- 72.

Ídem (2001), Los siete saberes necesarios para la educación del futuro. Paidós, Barcelona, Paris.

Naranjo, C. (2008), "La educación que tenemos roba a los jóvenes, la conciencia, el tiempo y la vida”. Revista Namaste.

Ídem (2010), Cambiar la educación para cambiar el mundo. Ed. La Llave, Vitoria.

Naredo, J. M. (1987), La economía en evolución. Historia y perspectivas de las categorías básicas del pensamiento económico. Ministerio de Economía y Hacienda, Secretaría de Estado y Comercio, Siglo XXI, Madrid.

Ortega y Gasset, J. (1930), Misión de la Universidad. Alianza Editorial, Madrid. (Ed. 1997).

González, C. (2013), Veintitrés maestros con corazón. Un salto cuántico en la enseñanza. (Consultado en febrero 2013,http:// sindamel.files.wordpress.com/2010/02/veintitresmaestrosdecorazon.pdf)

Pigem, J. (2010), GPS (Global, Personal, Social). Valores para un mundo 
Polis, Revista Latinoamericana, Volumen 12, No 35, 2013

en transformación. Ed. Kairós, Barcelona

Prigogine, I. (1983), ¿Tan sólo una ilusión? Una exploración del caos al orden.Tusquets Ed., Barcelona (Ed. 1997).

Prigogine, I. y Stengers, I. (1983), La nueva alianza. Metamorfosis de la ciencia. Alianza Editorial (Ed. 2002)

Recibido: 08.01.2013

Aceptado: 16.02.2013 